\title{
Seleção de micro-organismos celulolíticos, comparados com o Trichoderma reesei CCT2768
}

\author{
Douglas Fernandes da Silva ${ }^{1,2}$, Douglas Fernandes da Silva ${ }^{1,2}$, Bianca Consorti \\ Bussamra $^{1}$, Rafaela Camargo dos Santos ${ }^{1}$, Ioana Bittencourt Mourão', Ana Flavia \\ Carvalho ${ }^{3}$, Pedro de Oliva Neto ${ }^{1}$
}

${ }^{1}$ Universidade Estadual Paulista - UNESP - Assis-SP, Departamento de Ciências Biológicas, ${ }^{2}$ Universidade Estadual Paulista - UNESP - Rio Claro-SP, Departamento de Bioquímica, ${ }^{3}$ Universidade Estadual de Campinas - UNICAMP - Faculdade de Engenharia de Alimentos. e-mail: douglasfsilva@gmail.com

\section{RESUMO}

As celulases atualmente são enzimas extensivamente estudadas para a hidrólise de resíduos lignocelulósicos para obtenção de açúcares fermentescíveis utilizáveis em diferentes processos biotecnológicos, como a produção de bioetanol. O objetivo deste trabalho foi encontrar fungos celulolíticos que sejam eficientes nos processos de degradação da biomassa lignocelulósica. No presente trabalho foram selecionados 4 fungos celuloliticos previamente isolados pelo Laboratório de Biotecnologia Industrial (UNESP - Assis). Estes fungos foram cultivados em bagaço de cana-de-açúcar como substrato e para seus extratos enzimáticos foram testados suas atividades para celulases (FPase) e endoglucanases (CMCase). Os fungos FS09 (0,054 FPU/mL e 1,79 FPU/g de substrato, 0,874 U/mL e 29,1 U/g de substrato) e M51 (0,049 FPU/mL e 1,62 FPU/g de substrato, $1,094 \mathrm{U} / \mathrm{mL}$ e $36,46 \mathrm{U} / \mathrm{g}$ de substrato), mostrando valores superiores ao fungo T.reesei CCT 2768 que é referência em estudos e produção de celulases.

Palavras-chave: Celulases, açúcares fermentescíveis, bagaço de cana-de-açúcar, T.reesei CCT 2768.

\section{INTRODUÇÃO}

A necessidade de suprimento de energia para a manutenção de toda a estrutura e dinâmica operacional da sociedade representa um ponto crucial nas mais diversas áreas, como no próprio bem-estar individual até nos bioprocessos industriais e de prestações de serviços. O aumento no uso de energias não renováveis, como o petróleo, acarretou, ao longo dos anos, transformações tanto econômicas, como sociais e ambientais. Desta forma, a sua escassez pelo uso prolongado, acarreta um aumento do valor destas fontes não renováveis de energia. Estimativas pessimistas afirmam que em torno de 40 anos, estes combustíveis, irão se esgotar ${ }^{1}$. Com isso, e somado ao risco de colapso ambiental, torna-se necessária a busca de fontes alternativas e renováveis de energia, as chamadas energias limpas, como o bioetanol e bioetanol de segunda geração ${ }^{2}$. Os resíduos lignocelulósicos atualmente estão sendo visados para a utilização em processos biotecnológicos devido a aspectos econômicos, ambientais e a farta disponibilidade ${ }^{2}$. Uma das aplicações destes resíduos é a produção de enzimas celulolíticas para uma futura obtenção de açúcares fermentescíveis, os quais podem ser utilizados para geração do bioetanol de segunda geração, e assim servir como fonte de energia renovável ${ }^{3}$. Tendo como consequência a redução da poluição. Na natureza existem diversos fungos que 


\section{Anais do III Simpósio de Bioquímica e Biotecnologia Trabalho Completo apresentado na seção: PÔSTER}

\section{IIISIMBBTEC}

podem hidrolisar essa biomassa lignocelulósica, como o Trichoderma reesel ${ }^{4}$, contudo inúmeros outros micro-organismos que possuem essa capacidade podem ser isolados, e estes produzirem enzimas do complexo celulolítico.

No atual contexto mundial mostra-se uma necessidade da aquisição de recursos práticos e viáveis economicamente para a obtenção de fontes de energias renováveis, e para o reaproveitamento dos resíduos lignocelulósicos. Desta forma, este trabalho teve como objetivo foi de selecionar fungos celulolíticos promissores, e destes realizar cultivos de forma econômica visando obter extrato enzimático com atividade celulolítica, igual ou superior ao obtido pelo fungo T.reesei $\mathrm{CCT} 2768$.

\section{MATERIAL E MÉTODOS}

As 5 linhagens fúngicas que foram utilizadas estão depositadas na coleção de culturas da Faculdade de Ciências e Letras de Assis - UNESP, em que 4 delas foram codificadas como: M51, MG10, FS09, FS11A e o Trichoderma reesei CCT 2768 como referência na produção de enzimas celulolíticas. Foi utilizado tampão Acetato $(0,2 \mathrm{~mol} / \mathrm{L}-\mathrm{pH} 5,6)$ e como substrato para dosagem da atividade hidrolítica, papel de filtro Whatman $\mathrm{n}^{\circ} 1$ para a técnica de FPase, e Carboximetilcelulose (Sigma) para a técnica de CMCase, e como substrato para o cultivo dos micro-organismos bagaço de cana in natura.

Os micro-organismos foram cultivados em tubos contendo $5 \mathrm{~mL}$ de meio PDA 39g/L (potato, dextrose e ágar) inclinado (slants). Após cinco dias os micro-organismos foram repicados para placas de Petri contendo aproximadamente $30 \mathrm{~mL}$ de meio: $3 \%(\mathrm{p} / \mathrm{v})$ de bagaço de cana, $0,4 \%$ de sulfato de amônia, $0,1 \%$ de fosfato de potássio e $0,5 \%$ de extrato de levedura, com adição de Agar na concentração de $20 \mathrm{~g} / \mathrm{L}$. Estas placas foram mantidas por sete dias em temperatura ambiente a fim de se obter um crescimento fúngico desejável, sendo em seguida transferidos para Erlenmeyers de $250 \mathrm{~mL}$ para fermentação e obtenção do extrato celulolítico, no qual continha $80 \mathrm{~mL}$ do meio descrito anteriormente, sem a adição do Agar. Os 4 micro-organismos isolados, mais o Trichoderma reesei CCT2768, foram fermentados por 5 dias, em pHs diferentes: 3,$5 ; 4,5$ e 5,5, temperaturas diferentes: 28,30 e $35^{\circ} \mathrm{C}$, à $180 \mathrm{rpm}$. No final dos cultivos e filtração do extrato fúngico, foram medidas as atividades celulolíticas pelas técnicas de FPase $^{5}$ e CMCase ${ }^{6}$ (Ghose, 1987).

\section{RESULTADOS E DISCUSSÃO}

As atividades enzimáticas volumétricas para a técnica de FPase $(F P U / m L)$ e para técnica de CMCase $(\mathrm{UI} / \mathrm{mL}$ ) dos extratos celulolíticos obtidos após a fermentação desses micro-organismos, e em todas as condições testadas, estão representadas abaixo, nas figuras 1 e 2.

Figura 1: As atividades enzimáticas para a técnica de FPase (FPU/mL) dos 4 micro-organismos previamente selecionado mais o T.reesei CCT 2768. 


\section{Anais do III Simpósio de Bioquímica e Biotecnologia Trabalho Completo apresentado na seção: PÔSTER}

\section{IIISIMBBTEC}

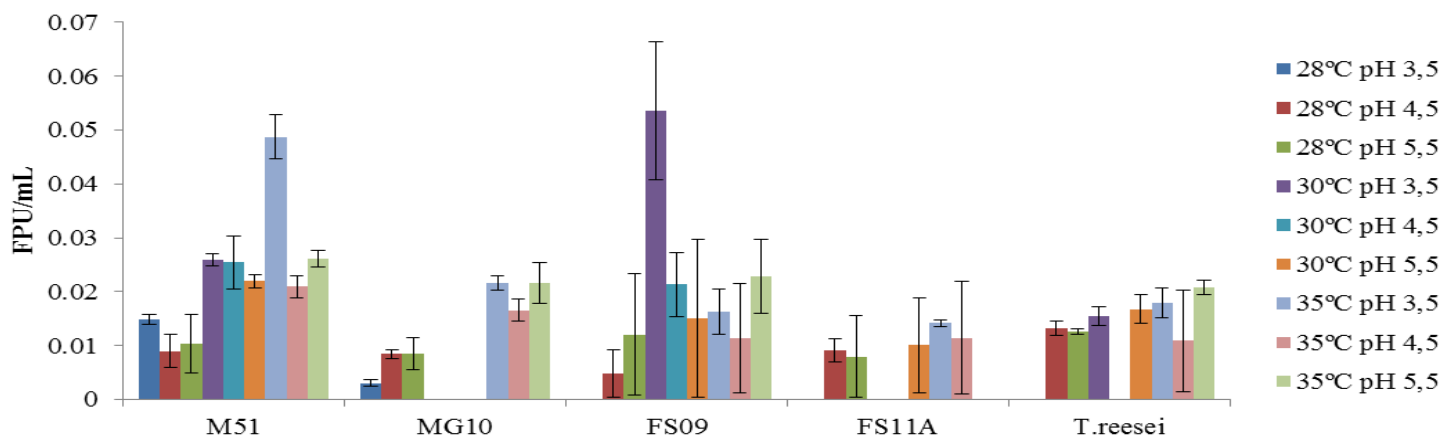

Figura 2: As atividades enzimáticas para a técnica de CMCase (UI/mL) dos 4 micro-organismos previamente selecionado mais o T.reesei CCT 2768.

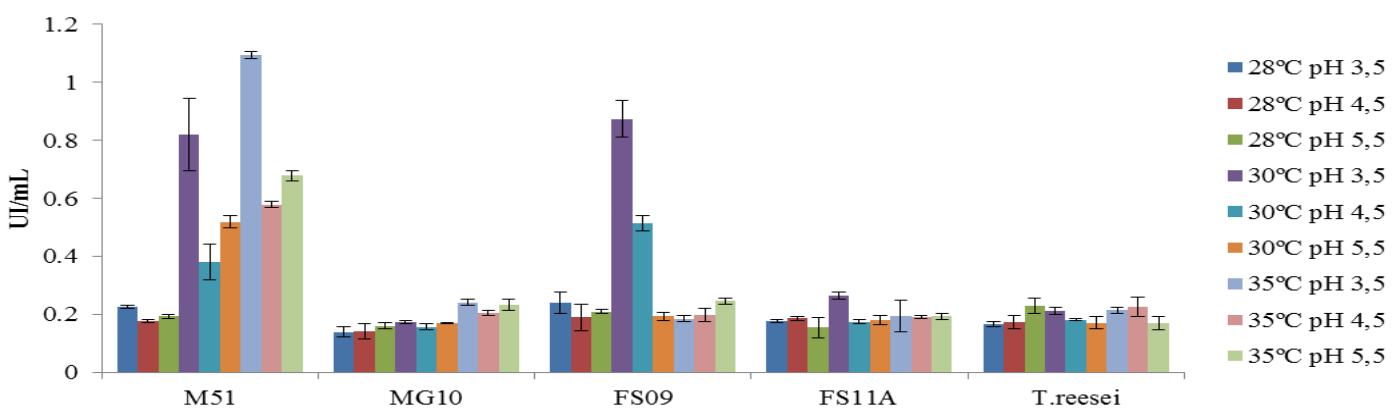

Como se pode observar o fungo que apresentou maior atividade enzimática para a técnica de FPase (figura 1) foi o FS09 com $0,054 \pm 0,01 \mathrm{FPU} / \mathrm{mL}$ em $30^{\circ} \mathrm{C}$ e pH 3,5, seguido pelo M51 com $0,049 \pm 0,004 \mathrm{FPU} / \mathrm{mL}$ em $35^{\circ} \mathrm{C}$ e $\mathrm{pH} 3,5$, porém sem diferença estatísticas (ANOVA $\mathrm{F}=15,83 \mathrm{e}$ Tukey $\mathrm{p}>0,05)$. Ambos foram muito superiores aos maiores valores do MG10A $(0,022 \pm 0,003$

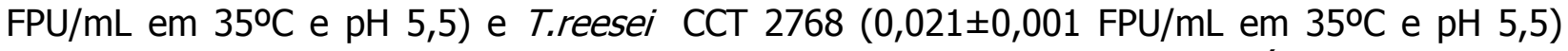
(ANOVA $\mathrm{F}=15,83$ e Tukey $\mathrm{p}<0,05$ ). Ao analisar as endoglucanases, pela técnica de CMCase (figura 2), observa-se que os melhores resultados foram para o fungo M51 com 1,094 $\pm 0,01$

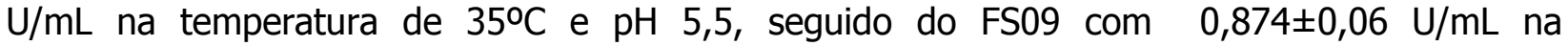
temperatura de $30^{\circ} \mathrm{C}$ e $\mathrm{pH} 3,5$, contudo desta vez, apesar de pequena a diferença entre eles, foi significante para $\mathrm{p}<0,01$ (ANOVA $\mathrm{F}=342,51$ ). $\mathrm{E}$, ambos os fungos apresentaram um valor muito superior ao maior valor de T.reesei CCT $2768\left(0,228 \pm 0,02 \mathrm{U} / \mathrm{mL}\right.$ em $28^{\circ} \mathrm{C}$ e pH 5,5) (ANOVA $\mathrm{F}=342,51$ e Tukey $\mathrm{p}<0,001)$. As atividades enzimáticas por grama de substrato em peso seco, para ambas as técnicas, FPase (FPU/g de substrato) e CMCase (U/g de substrato), estão apresentadas na Tabela 1. Esta forma de analisar a atividade celulolítica apresentada para os extratos fúngico desses micro-organismos, resulta em um rendimento mais real do processo de produção enzimática, já que é dado o potencial de produção destas enzimas por grama de substrato ofertado durante a fermentação, assim temos um valor mais verdadeiro dos possíveis custos para a produção deste complexo enzimático.

Tabela 1: Atividade Enzimática por grama de substrato (FPU/g substrato e U/g substrato) dos micro-organismos selecionados mais o T.reesei CCT 2768 - Fermentações a 28,30 e $35^{\circ} \mathrm{C}$, nos $\mathrm{pH} 3,5 ; 4,5$ e 5,5 . 


\section{Anais do III Simpósio de Bioquímica e Biotecnologia Trabalho Completo apresentado na seção: PÔSTER}

\section{IIISIMBBTEC}

\begin{tabular}{|c|c|c|c|c|c|c|c|c|c|c|c|c|c|c|c|c|c|c|}
\hline \multirow[b]{3}{*}{ Micror } & \multicolumn{6}{|c|}{$28^{\circ} \mathrm{C}$} & \multicolumn{6}{|c|}{$30^{\circ} \mathrm{C}$} & \multicolumn{6}{|c|}{$35^{\circ} \mathrm{C}$} \\
\hline & \multicolumn{2}{|c|}{ pH 3,5 } & \multicolumn{2}{|c|}{ pH 4,5 } & \multicolumn{2}{|c|}{ pH 5,5 } & \multicolumn{2}{|c|}{ pH 3,5 } & \multicolumn{2}{|c|}{ pH 4,5 } & \multicolumn{2}{|c|}{ pH 5,5 } & \multicolumn{2}{|c|}{ pH 3,5 } & \multicolumn{2}{|c|}{ pH 4,5 } & \multicolumn{2}{|c|}{ pH 5,5 } \\
\hline & $\begin{array}{l}F P U / g \\
\text { subst }\end{array}$ & $\begin{array}{c}\mathrm{U} / \mathrm{g} \\
\text { subst }\end{array}$ & $\begin{array}{l}F P U / g \\
\text { subst }\end{array}$ & $\begin{array}{c}U / g \\
\text { subst }\end{array}$ & $\begin{array}{l}F P U / g \\
\text { subst }\end{array}$ & $\begin{array}{c}U / g \\
\text { subst }\end{array}$ & $\begin{array}{c}F P U / g \\
\text { subst }\end{array}$ & $\begin{array}{c}U / g \\
\text { subst }\end{array}$ & $\begin{array}{l}F P U / g \\
\text { subst }\end{array}$ & $\begin{array}{c}U / g \\
\text { subst }\end{array}$ & $\begin{array}{l}F P U / g \\
\text { subst }\end{array}$ & $\begin{array}{c}U / g \\
\text { subst }\end{array}$ & $\begin{array}{l}\text { FPU/g } \\
\text { subst }\end{array}$ & $\begin{array}{c}U / g \\
\text { subst }\end{array}$ & $\begin{array}{l}\text { FPU/g } \\
\text { subst }\end{array}$ & $\begin{array}{c}U / g \\
\text { subst }\end{array}$ & $\begin{array}{c}F P U / g \\
\text { subst }\end{array}$ & $\begin{array}{c}U / g \\
\text { subst }\end{array}$ \\
\hline $\boldsymbol{M}$ & 0,49 & 7,56 & 0,38 & 5,89 & 0,61 & 6,43 & 0,86 & 27,37 & 0,85 & 12,70 & 0,73 & 17,28 & 1,62 & 36,47 & 0,70 & 19,27 & 0,87 & 22,62 \\
\hline MG10 & 0,32 & 4,66 & 0,40 & 4,71 & 0,38 & 5,35 & 0,00 & 5,77 & 0,00 & 5,25 & 0,00 & 5,66 & 0,72 & 8,06 & 0,55 & 6,83 & 0,72 & 7,77 \\
\hline FSO9 & 0,00 & 8,00 & 0,09 & 6,33 & 0,40 & 6,97 & 1,79 & 29,13 & 0,71 & 17,16 & 0,49 & 6,48 & 0,54 & 6,19 & 0,38 & 6,60 & 0,76 & 8,24 \\
\hline FS11A & 0,00 & 5,89 & 0,41 & 6,23 & 0,15 & 5,15 & 0,00 & 8,83 & 0,00 & 5,83 & 0,33 & 6,01 & 0,47 & 6,48 & 0,38 & 6,36 & 0,00 & 6,48 \\
\hline T.reesei & 0,00 & 5,59 & 0,68 & 5,74 & 0,42 & 7,61 & 0,51 & 7,07 & 0,00 & 6,07 & 0,56 & 5,72 & 0,60 & 7,18 & 0,36 & 7,54 & 0,69 & 5,66 \\
\hline
\end{tabular}

As atividades celulolíticas por grama de substrato medida pela técnica de FPase foram mais elevadas nos extratos produzidos pelos fungos FS09 $(1,79 \pm 0,042 \mathrm{FPU} / \mathrm{g}$ de substrato) e M51 $(1,62 \pm 0,14 \mathrm{FPU} / \mathrm{g}$ de substrato), sendo muito superior a apresentada pelo $T$. reesei CCT 2768 $(0,069 \pm 0,04 \mathrm{FPU} / \mathrm{g}$ de substrato) (ANOVA $\mathrm{F}=15,19$ e Tukey $\mathrm{p}<0,05)$. No caso da CMCase, 0 fungo $\mathrm{M} 51$ obteve $36,46 \pm 0,37 \mathrm{U} / \mathrm{g}$ de substrato, mostrando-se neste caso superior ao o FS09 com 29,13 $\pm 2,12 \mathrm{U} / \mathrm{g}$ de substrato, (ANOVA $\mathrm{F}=373,35$ e Tukey $\mathrm{p}<0,01$ ). De uma forma geral, os resultados obtidos neste trabalho revelaram-se promissores, dado que os parâmetros operacionais relevantes no processo de incubação como temperatura, aeração, formulação do meio, tempo de fermentação e até um possível pré-tratamento do substrato poderão ainda serem otimizados.

\section{CONCLUSÕES}

A partir do cultivo em meio de cultura a base de bagaço de cana foi possível selecionar linhagens de fungos filamentosos com maior atividade celulolítica do que o Trichoderma reesei CCT 2768. O destaque foi para os fungos ainda não identificados denominados FS09 e M51. As condições de $\mathrm{pH}$ e temperatura de cultivo influenciaram significativamente na produção enzimática com destaque para o pH 3,5 e temperaturas de 30 e $35^{\circ} \mathrm{C}$.

\section{REFERÊNCIAS}

(1) ODAC - The Oil Depletion Analysis Centre. Disponível em www.odac-info.org. Acesso em maio de 2013.

(2) PEREIRA JR., N.; COUTO, M.A.P.G.; SANTA ANNA, L.M.M. BIOMASS OF LIGNOCELLULOSIC COMPOSITION FOR FUEL ETHANOL PRODUCTION AND THE CONTEXT OF BIOREFINERY. IN SERIES ON BIOTECHNOLOGY, ED. AMIGA DIGITAL UFRJ, RIO DE JANEIRO, V.2, 45 P, 2008.

(3) DASHTBAN, M.; MAKI, M.; LEUNG, K.T.; MAO, C.; QIN, W. Cellulase activities in biomass conversion: measurement methods and comparison. Critical Review in Biotechnology, v.30, n.4, p.302-309, 2010.

(4) FUJII, T.; MURAKAMI, K; SAWAYANA, S. Cellulase Hyperproducing Mutants Derived from the Fungus Trichoderma reesei QM9414 Produced Large Amounts of Cellulase at the Enzymatic and Transcriptional Levels. Biomass Technology Research Center, Nacional Institute Of Advanced Industrial Science and Technology (AIST), 2-2-2 Hiro-suehiro, Kure, Hiroshima. Japan, 2010.

(5) MANDELS, M.; ANDREOTTI, R.E.; ROCHE, C. Measurement of scarifying cellulose.

Biotechnol. Bioeng. Symp., v.6, p.1471, 1976.

(6) GHOSE, T.K. Measurement of cellulose activities. Pure Appl Chem, v.59, p.257-268, 1987. 